Subscriber access provided by Caltech Library Services

Communication

\title{
Exceptional resilience of small-scale Au30Cu25Zn45 under cyclic stress-induced phase transformation
}

Xiaoyue Ni, Julia R Greer, Kaushik Bhattacharya, Richard D. James, and Xian Chen

Nano Lett., Just Accepted Manuscript • DOI: 10.1021/acs.nanolett.6b03555 • Publication Date (Web): 04 Nov 2016

Downloaded from http://pubs.acs.org on November 7, 2016

\section{Just Accepted}

"Just Accepted" manuscripts have been peer-reviewed and accepted for publication. They are posted online prior to technical editing, formatting for publication and author proofing. The American Chemical Society provides "Just Accepted" as a free service to the research community to expedite the dissemination of scientific material as soon as possible after acceptance. "Just Accepted" manuscripts appear in full in PDF format accompanied by an HTML abstract. "Just Accepted" manuscripts have been fully peer reviewed, but should not be considered the official version of record. They are accessible to all readers and citable by the Digital Object Identifier (DOI®). "Just Accepted" is an optional service offered to authors. Therefore, the "Just Accepted" Web site may not include all articles that will be published in the journal. After a manuscript is technically edited and formatted, it will be removed from the "Just Accepted" Web site and published as an ASAP article. Note that technical editing may introduce minor changes to the manuscript text and/or graphics which could affect content, and all legal disclaimers and ethical guidelines that apply to the journal pertain. ACS cannot be held responsible for errors or consequences arising from the use of information contained in these "Just Accepted" manuscripts. 


\title{
Exceptional resilience of small-scale $\mathbf{A} \mathbf{u}_{30} \mathbf{C u}_{25} \mathbf{Z n}_{45}$ under cyclic stress-induced phase transformation
}

\author{
Xiaoyue Ni, ${ }^{\dagger}$ Julia R. Greer, ${ }^{\dagger}$ Kaushik Bhattacharya, ${ }^{\dagger}$ Richard D. James, ${ }^{\ddagger}$ and \\ Xian Chen ${ }^{*}$, \\ $\dagger$ Division of Engineering and Applied Science, California Institute of Technology, \\ Pasadena, CA 91125 USA \\ $\ddagger$ Department of Aerospace Engineering and Mechanics, University of Minnesota, \\ Minneapolis, MN 55455 USA \\ \Department of Mechanical and Aerospace Engineering, Hong Kong University of Science \\ and Technology, Hong Kong \\ E-mail: xianchen@ust.hk
}

\begin{abstract}
Shape memory alloys that produce and recover from large deformation driven by martensitic transformation are widely exploited in biomedical devices and microactuators. Generally their actuation work degrades significantly within first a few cycles, and is reduced at smaller dimensions. Further, alloys exhibiting unprecedented reversibility have relatively small superelastic strain, $0.7 \%$. These raise the questions of whether high reversibility is necessarily accompanied by small work and strain, and whether high work and strain is necessarily diminished at small scale. Here we conclusively demonstrate that these are not true by showing that $\mathrm{Au}_{30} \mathrm{Cu}_{25} \mathrm{Zn}_{45}$ pillars exhibit $12 \mathrm{MJ} \mathrm{m}^{-3}$ work and 3.5\% superelastic strain even after 100,000 phase transformation cycles. Our findings confirm that the lattice compatibility dominates the


mechanical behavior of phase-changing materials at nano to micron scales, and points a way for smart micro-actuators design having the mutual benefits of high actuation work and long lifetime.

\section{Keywords}

Nanomechanics, nano- and micro-actuation, in-situ nanocompression

By far, the most successful application of shape memory alloys (SMA) is the stent, ${ }^{1,2}$ i.e. the expandable tube used to treat narrowed or weakened arteries in the human's body. This application only requires one-time stress-induced phase transformation so that the tube can be easily squeezed into a tiny radius and stand in place after the removal of stress. There are many patents and demonstrations for nano- and micro-actuation applications using SMA, ${ }^{3,4}$ but the functional degradation of general SMA upon cyclic phase transformations ${ }^{5-7}$ strongly hinders such applications in reality. Recent advances in shape memory alloys $^{8,9}$ show that small thermal hysteresis and high mechanical fatigue correlate closely with the satisfaction of the cofactor conditions, ${ }^{10}$ i.e., conditions on lattice parameters that enable the formation of various elastically compatible microstructures during phase transformation. These conditions can be achieved by doping and tuning compositional variables. Chluba et $a l^{9}$ have demonstrated a $\mathrm{Co} / \mathrm{Cu}$ doped NiTi-based SMA family in which $\mathrm{Ti}_{54} \mathrm{Ni}_{34} \mathrm{Cu}_{12}$ and $\mathrm{Ti}_{54.7} \mathrm{Ni}_{30.7} \mathrm{Cu}_{12.3} \mathrm{Co}_{2.3}$ thin films show ultra-low mechanical fatigue properties over millions of full transformation cycles. Compared with their nearby compositions, the lattice parameters of these alloys satisfy the cofactor conditions closely. The alloy $\mathrm{Ti}_{54} \mathrm{Ni}_{34} \mathrm{Cu}_{12}$ presented nanoprecipitates of $\mathrm{Ti}_{2} \mathrm{Ni} .{ }^{11}$ These were theorized to contribute to the exceptional reversibility: the compatible austenite/martensite interfaces that follow from the cofactor conditions ${ }^{10}$ are also approximately parallel to the interfaces of the coherent precipitates. ${ }^{11}$ Using a similar development strategy, the bulk SMA $\mathrm{Au}_{30} \mathrm{Cu}_{25} \mathrm{Zn}_{45}{ }^{8}$ was found to satisfy the cofactor conditions for multiple twin systems. Thermal cycling tests on this alloy showed a nearly 
zero-migration in transition temperature and latent heat, as well as $<2^{\circ}$ thermal hysteresis for 16,000 thermal cycles. An important future application area for SMA is nano- to microscale actuation. ${ }^{2,3,12}$ Thus, it is particularly interesting to investigate whether the formation of abundant compatible microstructures under the cofactor conditions has implications for work output and reversibility in the small-scale regime.

Previous in-situ synchrotron X-ray Laue microdiffraction ( $\mu$ SXRD) analysis of $\mathrm{Au}_{30} \mathrm{Cu}_{25^{-}}$ $\mathrm{Zn}_{45}$ confirmed the fact that the phase transformation of this alloy between $\mathrm{L} 2_{1}$ austenite and the $\mathrm{P} 2{ }_{1}$ martensite is not accompanied by the formation of intermetallic precipitates. ${ }^{13}$ The lattice parameters closely satisfy the cofactor conditions for a family of $<100>$ compound twins and $<110>$ type I/II twins simultaneously. Quantitative characterization of microstructures at phase transformation revealed the elimination of elastic transition layers between austenite and single or multiple-twinned martensite variants. ${ }^{13}$ In this work, we utilize the geometrically nonlinear theory of martensite and nanomechanical experiments to investigate the cyclic mechanical behavior of $1 \mu \mathrm{m}$ - to $2 \mu \mathrm{m}$-diameter cylindrical pillars carved from a single austenite grain of $\mathrm{Au}_{30} \mathrm{Cu}_{25} \mathrm{Zn}_{45}$ plate using Focused Ion Beam (FIB) milling. These were subject to uniaxial compressive loading. The crystallographic orientation of the resulting pillars deviated from $[001]_{L 2_{1}}$ by $11.25^{\circ}$ (see Figure S2 in supplementary materials). Two types of experiments were conducted: (1) ex-situ cyclic compression tests in a nanoindenter equipped with a custom-made $8 \mu \mathrm{m}$-diameter diamond tip (TriboIndenter, Hysitron, Inc.) and (2) in-situ compression with a $2 \mu$ m-diameter diamond tip built in a custommade instrument comprised of a nanoindenter-like module (PI 85 PicoIndenter, Hysitron, Inc.) inside of a DualBeam FIB (Versa 3D, FEI) that permits in-situ video recording and imaging.

Figure 1 demonstrates the results from the cyclic compression tests conducted in the $e x$ situ TriboIndenter on the sample with $\sim 2 \mu \mathrm{m}$ diameter and $\sim 6 \mu \mathrm{m}$ height. At each cycle $10^{n}$, $n=0,1,2, \ldots, 5$, the force-displacement response was acquired for a full phase transformation cycle by quasi-static displacement control, i.e., load up to the elastic regime of martensite and 


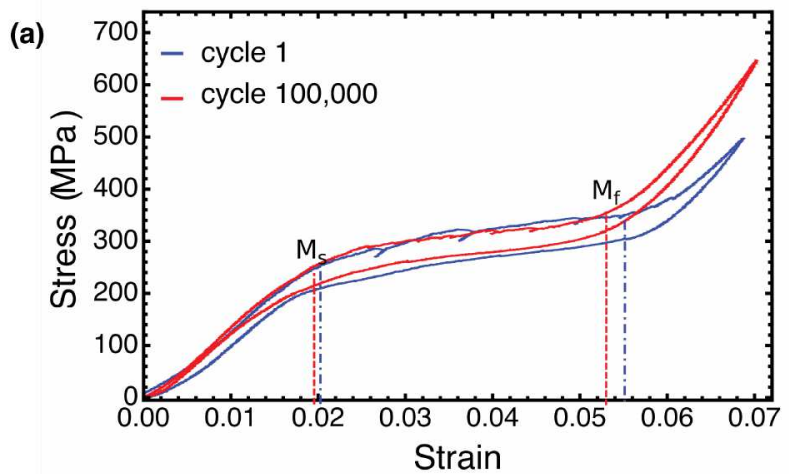

(b)
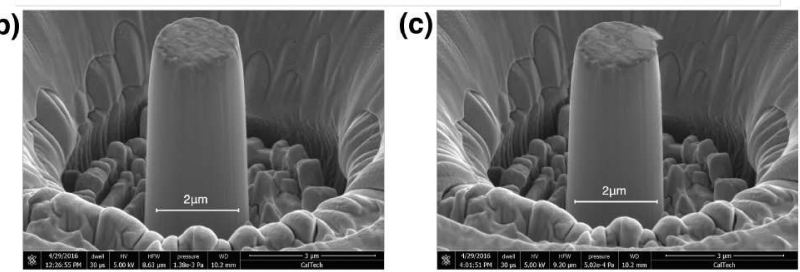

(d)

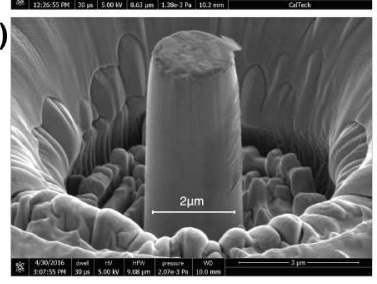

(e)

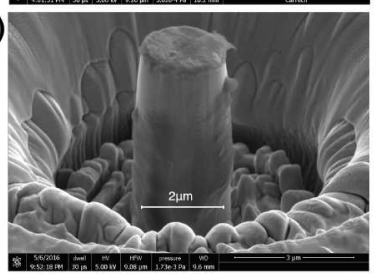

Figure 1: The $\mathrm{Au}_{30} \mathrm{Cu}_{25} \mathrm{Zn}_{45}$ shape memory $2 \mu$ m-diameter pillar subjected to 100,000 compression-induced phase transformation cycles. (a) Stress vs. strain data for the first and last cycles of the $2 \mu$ m-diameter pillar. $M_{s}$ and $M_{f}$ mark the martensite start / finish states respectively. (b) - (e) Post-mortem SEM images of the $2 \mu$ m-diameter pillar after cycle $1,1,000,10,000$ and 100,000 respectively. 
unload down to undeformed austenite. The force-displacement data was converted to the true stress-strain curve using the procedure outlined in reference. ${ }^{14}$ The result is shown in Figure 1 (a) for the 1st cycle (blue) and 100,000th cycle (red). The superelastic plateau strain, defined as the difference between strains at the states of martensite start/finish, marked as $M_{s}$ and $M_{f}$ in Figure $1(\mathrm{a})$, is $\sim 3.7 \%$ for cycle 1 and $\sim 3.5 \%$ for cycle 100,000, both corresponding to about $300 \mathrm{MPa}$ transformation stress. At the end of the 100,000th cycle, nearly $7 \%$ total strain at $800 \mathrm{MPa}$ peak stress was completely recovered upon unloading. The SEM images of post-mortem samples at the end of the 1st, 1,000th, 10,000th and 100,000th cycles are shown in Figure 1 (b) - (e). These images show the formation of a thin carbon layer on the sample surfaces, typical for performing experiments in vacuum chambers of electron microscopes, which started to peel off at higher cycle numbers. No signs of permanent deformation nor structural damage of the sample were observed at any point during the experiments.

We define the one-way work, $W$, as the area underneath the stress $(\sigma)$ - strain $(\epsilon)$ curve between $M_{s}$ and $M_{f}$ states during the compression-induced martensitic transformation,

$$
W=\int_{M_{s}}^{M_{f}} \sigma(\epsilon) d \epsilon
$$

Figure 2 presents the one-way work calculated using Eq. (1) for $1 \mu \mathrm{m}$ - and $2 \mu \mathrm{m}$-diameter $\mathrm{Au}_{30} \mathrm{Cu}_{25} \mathrm{Zn}_{45}$ pillars of the same crystallographic orientation. It reveals a subtle size effect that the $1 \mu \mathrm{m}$ pillar has less work than the $2 \mu \mathrm{m}$ pillar. The average work over 100,000 cycles is $\sim 10 \mathrm{MJ} \mathrm{m}^{-3}$ for $1 \mu \mathrm{m}$ pillar and $\sim 12 \mathrm{MJ} \mathrm{m}^{-3}$ for $2 \mu \mathrm{m}$ pillar, either of which is among the largest values of one-way work used for modern actuation systems, ${ }^{15-19}$ and is comparable to the bulk NiTi, ${ }^{5,20}$ i.e., the most successful SMA exploited for actuation systems and selfexpandable stents. ${ }^{2,15,17}$ In contrast to bulk NiTi, which at comparable stresses loses nearly half of its work within only 100 cycles, ${ }^{5,21,22}$ these micrometer structures retain their large actuation work over 100,000 cycles. $4 \sim 5 \%$ one-way superelastic strain were also observed in some shape memory alloys such as ferromagnetic SMAs $\mathrm{Ni}_{2} \mathrm{MnX}(\mathrm{X}=\mathrm{In}, \mathrm{Sn}, \mathrm{Ga})$ and 


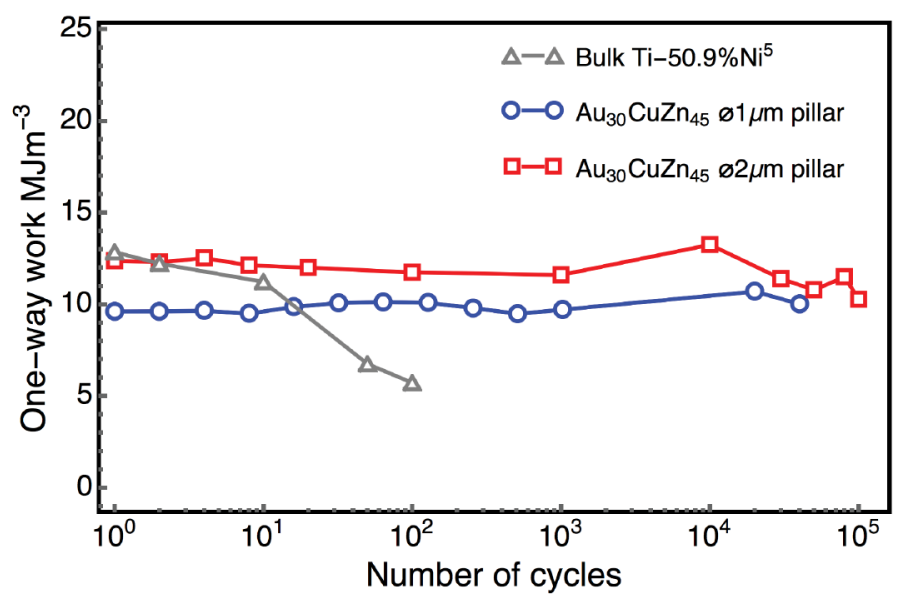

Figure 2: Comparison of cyclic degradation between nearly equal-atomic NiTi (Miyazaki et al $(1986)^{5}$ ) and $\mathrm{Au}_{30} \mathrm{Cu}_{25} \mathrm{Zn}_{45}$ micrometer pillars (this work). The work is calculated using Eq. (1) as the area under the stress-strain curve between $M_{s}$ and $M_{f}$ shown in Figure 1(a).

Cu-based SMAs, however the phase transformations in these alloys were driven in much lower stress, i.e. $<50 \mathrm{MPa}^{23,24}$ for ferromagnetic SMAs and $\sim 100 \mathrm{MPa}$ for most Cu-based SMAs. ${ }^{6,16}$

We have characterized the superelasticity phenomenon related to martensitic transformation by burst events, defined as sudden jumps in force-displacement response, ${ }^{25}$ which varies from cycle to cycle in these samples. This is consistent with the irreproducibility of formation of martensite microstructure observed in bulk. ${ }^{8,13}$ However, the total work remained virtually the same in each cycle. Normally, in uniaxial compression experiments on martensitic pillars, a single shear band with sharp re-entrant corners forms, ${ }^{18,26,27}$ leading to strong stress concentrations. Despite of the large superelastic plateau strain delivered by the pillars studied in this work, their lateral surfaces remain smooth (see the in-situ movies S1 and S2). Evidently, satisfaction of the cofactor conditions permits numerous low and zero elastic energy nanostructures exhibiting quite smooth deformations even at such a small scale.

In cubic to monoclinic transformations, there are 12 distinct martensite variants with stretch tensors relative to austenite, related by the point group of austenite $\left(\mathcal{P}^{24}\right): \mathcal{M}=$ $\left\{\mathbf{U}_{1}, \ldots, \mathbf{U}_{12}\right\}=\left\{\mathbf{Q}_{i} \mathbf{U Q}_{i}^{T}: \mathbf{Q}_{i} \in \mathcal{P}^{24}\right\} .{ }^{28,29}$ According to energy minimization, the specific 
variants of martensite that form upon loading depend on the crystal orientation of the austenite micro-pillar and on the mechanical loading conditions. We characterized the end-surface normal of the micro-pillars by synchrotron Laue diffraction to be $\hat{\mathbf{N}}=(0.150,-0.125,0.981)$ that is close to a high symmetry direction $(0,0,1)$ with a slight angular deviation $11.25^{\circ}$. Such a near high symmetry orientation gives rise to four variants which approximately minimize the total free energy (see derivation in supplementary materials). If we assume that only one of these four variants nucleates and grows from the austenite in each of the loading cycles, the resultant compressive strain will be $4.7 \%$, which corresponds to the two shear strains of $7 \%$ and $4 \%$ determined by the crystallographic equations of martensite. ${ }^{28,29}$ However, the measured plateau strain, $M_{f}-M_{s}=3.5 \%$, in Figure 1 (a) is significantly lower than the compressive strain calculated from a single variant of martensite, which implies the existence of multiple martensite variants. Although a multi-variant microstructure may compromise the compressive strain, it better accommodates the loading device, which tends to favor neither bending nor shear of the pillar. The satisfaction of the cofactor conditions facilitates this process by allowing for a plethora of elastically compatible austenite/ twinned martensite structures.

To examine this process in more detail, we utilized a custom-made in-situ nanomechanical loading module to observe the formation of martensite inside the electron microscope while simultaneously performing the compression tests. Figure 3 shows the SEM images for another $\sim 2 \mu \mathrm{m}$-diameter pillar sample at $0 \%$ and $5 \%$ compressive strain (the in-situ deformation due to stress-induced phase transformation is shown in movies S1 and S2). This pillar underwent a significant deformation, $\sim 7 \%$, including elastic deformation and phase transformation, yet its surface remained smooth and did not exhibit large lateral shear. The SEM image in Figure $3(\mathrm{~d})$, which shows parallel and un-equally distributed wavy-patterns of the transformed pillar, is consistent with twinning having varying volume fractions. This microstructure is distinct from what has been observed in ordinary shape memory single/poly- crystals under uniaxial loading, ${ }^{21,22,26,30}$ and from the microstructure of nano- and micro-pillars deformed 

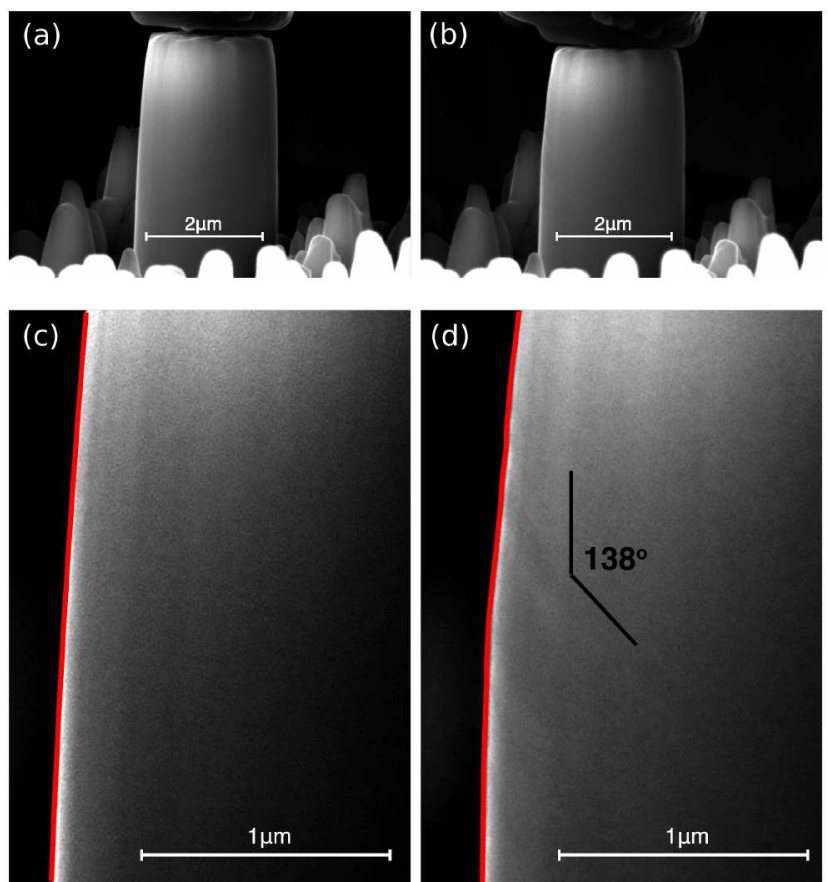

Figure 3: SEM images of the $2 \mu \mathrm{m}$-diameter pillar under in-situ nanomechanical experiments. (a) and (c) are the undeformed austenite phase; (b) and (d) are the 5\% strained martensite phase;

plastically by the motion of dislocations. ${ }^{14,31}$

We chose the variant giving the biggest compressive strain upon loading, by which we constructed a (101) type I twin system. Based on the geometrically nonlinear theory of martensite, ${ }^{10}$ we postulated a homogenous deformation that maps the austenite phase, shown Figure 4 (a), to the deformed martensite phase consisting of the (101) type I twin lamellae with varying volume fractions, shown in Figure 4 (b). The average deformation gradient of a $\hat{\mathbf{N}}$-oriented pillar with 1:3 aspect ratio results in a $3.2 \%$ compressive strain and a $1.8 \%$ shear strain. Compared to the shear strain caused by forming a single variant, the presence of the compatible (101) type I twin reduces the shear deformation by a large margin. In addition, the calculated compressive strain agrees with the plateau strain (Figure 1(a)) measured from the ex-situ nanocompression experiments. The austenite and martensite interface normals are calculated from the crystallographic equation of martensite (see Supplement), $\mathbf{m}_{0}=$ $(0.742,0.092,-0.665)$ for the blue variant and $\mathbf{m}_{1}=(-0.665,0.092,0.742)$ for the green variant. The formation of these twin lamellae matches the martensite morphology observed 
from in-situ nanomechanical experiments. The angle between the trace of interface and $\hat{\mathbf{N}}$ is $\sim 142^{\circ}$ shown in Figure 4(b), compared to the angle $\sim 138^{\circ}$ measured from the SEM image in Figure 3(d). Under the cofactor conditions satisfied by this alloy, it is possible to have untwinned austenite-martensite interfaces that are nearly parallel to a family of twin boundaries which, in fact, are those favored by the Schmid law. Normally, the scale of microstructure in a martensitic material is a consequence of the balance between the energy of stressed transition layers and the total interfacial energy on twin boundaries. However, in the microstructure calculated in Figure 4 there are theoretically no stressed transition layers and, therefore, with only the penalty of interfacial energy, quite complex microstructures are possible even below $1 \mu \mathrm{m}$ scale. As shown in Figure 3, these microstructures can form without sharp re-entrant corners and with volume fractions of both martensite variants and of austenite/martensite that vary smoothly with the loading condition.

In summary, the alloy $\mathrm{Au}_{30} \mathrm{Cu}_{25} \mathrm{Zn}_{45}$, which closely satisfies the cofactor conditions, exhibits unprecedented levels of work and reversibility in nanomechanical experiments. The analysis suggests that this is due to the presence of numerous compatible austenite/twinned martensite structures.As a consequence, the pillar can deform pseudo-homogeneously, even at micron scale, by using twinned nanostructures. The results may inspire the design of small-scale superelastic and actuation devices for which high levels of work and reversibility are particularly important.

\section{Acknowledgement}

XC acknowledge the financial support of the HK Research Grants Council through Early Career Scheme under Grant No. 26200316 and UGC Fund B002-0172-R9358. XN and JRG acknowledge the financial support of the U.S. Department of Energy through Early Career Research Program under Grant No. DE-SC0006599. KB and RDJ acknowledge the financial support of the Air Force Office of Scientific Research through MURI Grant 
(a)

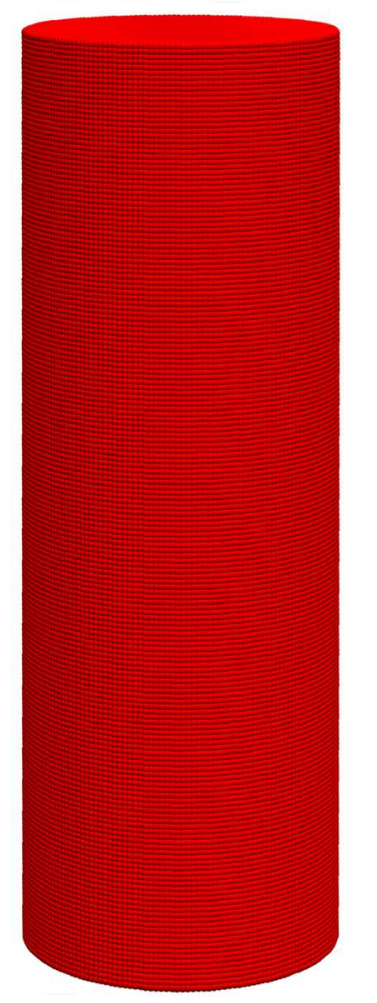

(b)

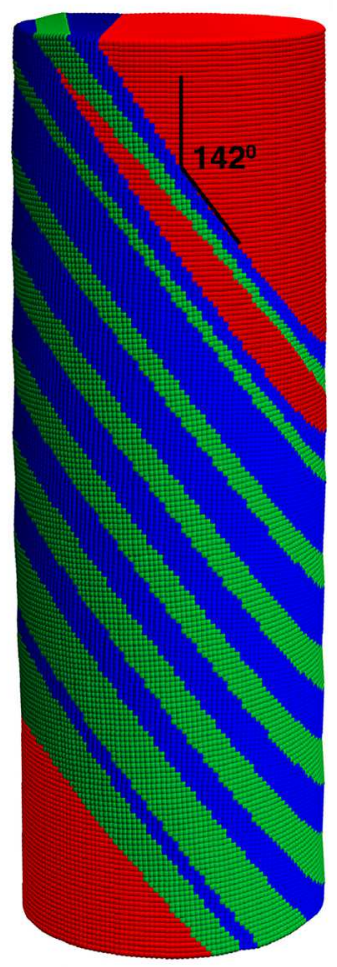

Figure 4: Formation of microstructure under uniaxial compressive loading predicted by the geometrically nonlinear theory of martensite. (a) Undeformed austenite phase. (b) Twinned martensite satisfying the cofactor conditions subject to the uniaxial loading. 
No. FA9550-12-1-0458. The research of RDJ was also supported by NSF-PIRE (OISE0967140), MURI (W911NF-07-1-0410 administered by AFOSR), ONR (N00014-14-1-0714), NSF-DMREF 1629160, the RDF Fund of the Institute on the Environment (UMN) and AFOSR (FA9550-15-1-0207). The Advanced Light Source is supported by the Director, Office of Science, Office of Basic Energy Sciences, of the U.S. Department of Energy under Contract No. DE-AC02-05CH11231.

\section{Supporting Information Available}

Details of the methods for cyclic nanomechanical experiments and the theoretical calculation of the formation of microstructure are given in the supplementary document. The movies showing the in-situ nanocompression are available from the following

- Filename: Movies S1

- Filename: Movies S2

This material is available free of charge via the Internet at http://pubs.acs.org/.

\section{References}

(1) Flomenblit, J.; Budigina, N.; Bromberg, Y. Two way shape memory alloy medical stent. US Patent 5562641 A, 1994.

(2) Zahn, J. D. In Bio-MEMS; Wang, W., Soper, S. A., Eds.; CRC Press: Boca Raton, Florida 33487, United States, 2007; Chapter 6, pp 143-176.

(3) Busch, J. D.; Johnson, A. D. Shape-memory alloy micro-actuator. US Patent 5061914 A, 1991.

(4) Kahn, H.; Huff, M. A.; Heuer, A. H. J. Micromech. Microeng. 1998, 8, 213-221. 
(5) Miyazaki, S.; Imai, T.; Igo, Y.; Otsuka, K. Metall. Trans. A 1986, 17A, 115-120.

(6) San Juan, J.; No, M. L.; Schuh, C. A. Adv. Mater. 2007, 20, 272-278.

(7) Bechtold, C.; Chluba, C.; de Miranda, R. L.; Quandt, E. Appl. Phys. Lett. 2012, 101, 091903.

(8) Song, Y.; Chen, X.; Dabade, V.; Shield, T. W.; James, R. D. Nature 2013, 502, 85-88.

(9) Chluba, C.; Ge, W.; Lima de Miranda, R.; Strobel, J.; Kienle, L.; Quandt, E.; Wuttig, M. Science 2015, 348, 1004-1007.

(10) Chen, X.; Srivastava, V.; Dabade, V.; James, R. D. J. Mech. Phys. Solids 2013, 61, $2566-2587$.

(11) Dankwort, T.; Strobel, J.; Chluba, C.; Ge, W.; Duppel, V.; Wuttig, M.; Quandt, E.; Kienle, L. J. Appl. Cryst. 2016, 49, 1009-1015.

(12) Bhattacharya, K.; James, R. D. Science 2005, 307, 53-54.

(13) Chen, X.; Tamura, N.; MacDowell, A.; James, R. D. Appl. Phys. Lett. 2016, 108, 211902.

(14) Greer, J.; Oliver, W. C.; Nix, W. D. Acta Mater. 2005, 53, $1821-1830$.

(15) Krulevitch, P.; Lee, A. P.; Ramsey, P. B.; Trevino, J. C.; Hamilton, J.; Northrup, M. A. J. Microelectromech. Syst. 1996, 5, 270-282.

(16) Sireday, N.; Eberhardt, A. Mater. Sci. Eng., A 2000, 290, 171-179.

(17) Bell, D. J.; Lu, T. J.; Fleck, N. A.; Spearing, S. M. J. Micromech. Microeng. 2005, 15, $153-164$.

(18) Ibarra, A.; San Juan, J.; Bocanegra, E. H.; Nó, M. L. Acta Mater. 2007, 55, 4789-4798. 
(19) Sutou, Y. and Omori, T. and Kainuma, R. and Ishida, K., Mater. Sci. Tech. 2008, 24, 896-901.

(20) Orgeas, L.; Favier, D. Acta Mater. 1998, 46, 5579-5591.

(21) Pelton, A. R.; Huang, G. H.; Moine, P.; Sinclair, R. Mater. Sci. Eng., A 2012, 532, 130-138.

(22) Sun, Q. P.; Li, Z. Q. Int. J. Solids Struct. 2002, 39, 3797-3809.

(23) Karaman, I.; Karaca, H. E.; Basaran, B.; Lagoudas, D. C.; Chumlyakov, Y. I.; Maier, H. J. Scripta Mater. 2006, 55, 403-406.

(24) Xu, X.; Ito, W.; Umetsu, R. Y.; Kainuma, R.; Ishida, K. Appl. Phys. Lett. 2009, 95, 181905.

(25) San Juan, J.; No, M. L.; Schuh, C. A. Nature Nano. 2009, 4, 415-419.

(26) Ichinose, S.; Funatsu, Y.; Otsuka, K. Acta Metall. 1985, 33, 1613-1620.

(27) Brinsona, L. C.; Schmidtb, I.; Lammering, R. J. Mech. Phys. Solids 2004, 52, 19541571.

(28) Ball, J. M.; James, R. D. Arch. Ration. Mech. Anal. 1987, 100, 13-52.

(29) Bhattacharya, K. Microstructure of martensite: why it forms and how it gives rise to the shape-memory effect; Oxford series on materials modeling; Oxford University Press: Oxford OX26DP, UK, 2003.

(30) Frick, C. P.; Clark, B. G.; Orso, S.; Sonnweber-Ribic, P.; Arzt, E. Scripta Mater. 2008, $59,7-10$.

(31) Jennings, A. T.; Burek, M. J.; Greer, J. Phys. Rev. Lett. 2010, 104, 135503. 


\section{Graphical TOC Entry}

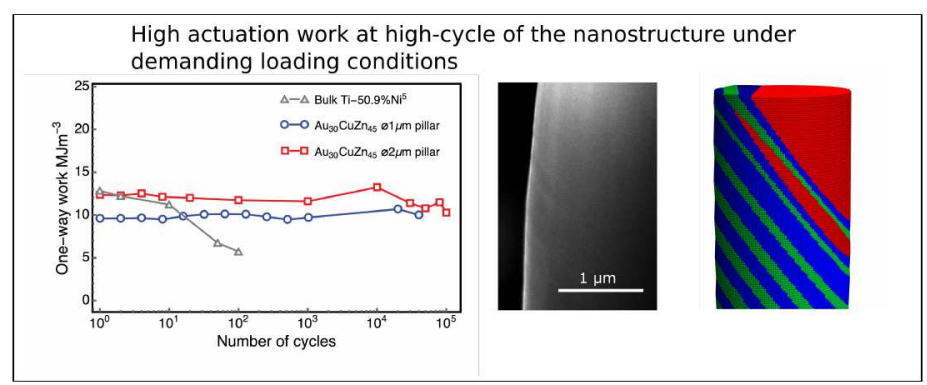

